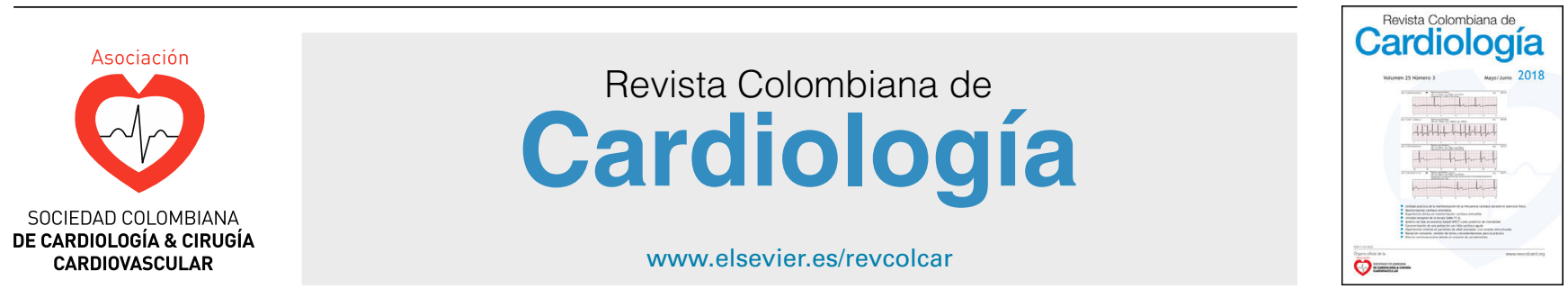

CARDIOLOGÍA DEL ADULTO - PRESENTACIÓN DE CASOS

\title{
Aortitis sifilítica: manifestación olvidada de la sífilis
}

\author{
Laura María Uribe-Arango ${ }^{a}$, Mateo Mejía-Zuluaga ${ }^{\mathrm{b}}$, \\ Tatiana María Bedoya-Jaramilloc ${ }^{c}$, Alejandro Arroyave-Carvajal ${ }^{\mathrm{d}, \mathrm{e}}$ \\ y Mauricio Duque-Ramírez ${ }^{\mathrm{f}, *}$
}

a Departamento de Medicina Interna, Universidad CES, Medellín, Colombia

b Fellow de Cardiología, Cardiología CES, Universidad CES, Medellín, Colombia

c Enlace Cardiología, CES Cardiología, Universidad CES, Medellín, Colombia

¿ Hospital General de Medellín, Medellín, Colombia

e Universidad CES, Medellín, Colombia

f Internista, Cardiólogo-Electrofisiólogo, director CES Cardiología, Universidad CES , Medellín, Colombia

Recibido el 11 de febrero de 2019; aceptado el 8 de mayo de 2019

Disponible en Internet el 2 de agosto de 2019

\section{PALABRAS CLAVE \\ Sífilis terciaria; \\ Sífilis cardiovascular; \\ Aortitis sifilítica; \\ Insuficiencia aórtica}

\section{KEYWORDS}

Tertiary syphilis;

Cardiovascular

syphilis;

Syphilitic aortitis;

Aortic valve failure;

Aortic aneurysm
Resumen La sífilis es una infección bacteriana producida por el Treponema pallidum (espiroqueta). Consta de varias etapas: primaria, secundaria y terciaria, según el tiempo de evolución desde el momento en que se adquiere la infección. Cada etapa comprende diferentes manifestaciones clínicas; los síntomas cardiovasculares forman parte de la sífilis terciaria, en cuyo caso la aortitis sifilítica es la principal forma de presentación.

Se expone el caso de un paciente que consultó por déficit neurológico focal, en quien por medio de estudios de extensión se documentó neurosífilis e insuficiencia valvular aórtica severa secundaria a perforación de la válvula coronaria derecha, que requirió recambio valvular aórtico por bioprótesis. Adicionalmente, se hace una revisión de las principales manifestaciones cardiovasculares de esta enfermedad.

Aunque en la era postantibiótica este tipo de manifestaciones tardías son cada vez menos frecuentes, es imperativo conocerlas.

๑ 2019 Publicado por Elsevier España, S.L.U. en nombre de Sociedad Colombiana de Cardiología y Cirugía Cardiovascular. Este es un artículo Open Access bajo la licencia CC BY-NC-ND (http:// creativecommons.org/licenses/by-nc-nd/4.0/).

\section{Syphilitic aortitis: forgotten manifestation of syphilis}

Abstract Syphilis is a bacterial infection caused by Treponema pallidum (spirochete). It has various stages: primary, secondary and tertiary; depending on the time to progression from the moment the infection is acquired. Each stage involves various clinical manifestations; cardiovascular symptoms are part of tertiary syphilis, and syphilitic aortitis is the main form of presentation.

\footnotetext{
* Autor para correspondencia.

Correo electrónico: mauricioduquemd@gmail.com (M. Duque-Ramírez).
} 
We present the case of a patient who consulted with a focal neurological deficit, in whom extension studies reported neurosyphilis and severe aortic failure secondary to perforation of the right coronary valve, which required aortic valve replacement by bioprosthesis. We also undertake a review of the main cardiovascular manifestations of this disease.

Although in the post-antibiotic era this type of late manifestation is increasingly less frequent, it is imperative that we are aware of it.

(c) 2019 Published by Elsevier España, S.L.U. on behalf of Sociedad Colombiana de Cardiología y Cirugía Cardiovascular. This is an open access article under the CC BY-NC-ND license (http:// creativecommons.org/licenses/by-nc-nd/4.0/).

\section{Introducción}

En lo concerniente a las enfermedades de transmisión sexual, la sífilis se destaca por su diversidad por cuanto sus manifestaciones clínicas, las cuales comprometen principalmente los sistemas tegumentario, neurológico y cardiovascular. Pese a la facilidad y amplia disponibilidad del tratamiento antibiótico con penicilina, la incidencia de infección por Treponema pallidum viene en aumento, posiblemente por su asociación con la infección del virus de la imunodeficiencia humana ${ }^{1}$. Las manifestaciones cardiovasculares, aunque poco reportadas en la literatura, ocurren hasta en el $70 \%$ de los casos con sífilis no tratada ${ }^{2-5}$; de ellas, la insuficiencia aórtica es la complicación más frecuente ${ }^{6}$. A continuación se expone el caso de un paciente masculino con neurolúes e insuficiencia aórtica severa por probable aortitis sifilítica.

\section{Caso}

Paciente masculino de 56 años, con antecedente de hipertensión arterial, quien consultó a una institución de tercer nivel de Medellín por cuadro clínico de tres días de evolución consistente en parestesias y hemiparesia del hemicuerpo derecho, asociado a disartria y asimetría facial; negaba síntomas cardiovasculares. Una tomografía de cráneo simple realizada a su ingreso, mostró área hipodensa en región gangliobasal izquierda compatible con lesión isquémica. Se amplió estudio para determinar etiología del infarto cerebral, encontrando VDRL reactivo en 128 dils y FTA-ABS positivo. En ecocardiografía transesofágica se evidenció válvula aórtica trivalva con insuficiencia severa, secundaria a perforación de la valva coronariana derecha, dilatación de la raíz aórtica con engrosamiento intimal difuso y calcificaciones en la unión sinotubular y en la curvatura menor del cayado aórtico (fig. 1). Se practicó arteriografía coronaria en la que se encontró dilatación de la aorta torácica ascendente con formación de aneurisma en el tercio medio del cayado aórtico y oclusión en el ostium de la arteria coronaria derecha. Una angiotomografía de tórax confirmó dilatación aneurismática de la aorta ascendente de $45 \mathrm{~mm}$ de diámetro (fig. 2). En vista de los hallazgos imagenológicos descritos en presencia de serología reactiva para sífilis, se consideró probable aortitis sifilítica. Se hizo recambio valvular aórtico exitoso, por bioprótesis y bypass aorto-coronario a la descendente posterior (fig. 3). El aneurisma de la aorta ascendente no cumplía criterios de diámetro que obligaran a su corrección. El estudio histológico del material quirúrgico arrojó cambios degenerativos; en la aorta se observó vasa vasorum con infiltrado inflamatorio de células mononucleadas, mientras que la tinción de Warthin-Starry fue negativa.

El análisis del líquido cefalorraquídeo mostró reactividad para VDRL en 4 dils, que ameritó cubrimiento antibiótico para neurolúes, con penicilina cristalina durante catorce días. Tuvo una evolución postoperatoria complicada por neumonía asociada al ventilador, choque séptico y falla multiorgánica que derivaron en su muerte.

\section{Discusión}

La sífilis es una infección bacteriana crónica producida por la espiroqueta Treponema pallidum, subespecie pallidum. Los humanos son su único huésped y su transmisión usualmente ocurre por contacto sexual ${ }^{6}$. Las manifestaciones cardiovasculares hacen parte de la sífilis terciaria, las cuales se presentan entre 5 y 30 años después de la infección inicial ${ }^{7}$. Aunque en la era postantibiótica este tipo de manifestaciones tardías son cada vez menos frecuentes, es obligatorio conocerlas, ya que se observan hasta en el $70 \%$ de los pacientes no tratados ${ }^{2-5}$. En Colombia para el año 2011 se reportaron 9.324 nuevos casos de sífilis, de los cuales el $10,6 \%$ correspondió a formas terciarias, y de éstos $1,5 \%$ a sífilis cardiovascular ${ }^{8}$.

La aortitis sifilítica es la principal manifestación de la sífilis cardiovascular; es secundaria a vasculitis de la vasa vasorum, la cual produce una endarteritis obliterante con daño isquémico de la túnica media de la aorta, al igual que pérdida del músculo liso y de la matriz extracelular ${ }^{9}$. En la mayoría de casos hay compromiso de la aorta torácica ascendente, debido a la vasa vasorum abundante y extensa red linfática de esta región, la cual es invadida por el Treponema pallidum durante la infección primaria. El compromiso exclusivo de la aorta torácica se debe a la ausencia de vasa vasorum en la aorta abdominal ${ }^{10}$. Por otra parte, el debilitamiento de la pared aórtica lleva a dilatación de la raiz aórtica y potenciales complicaciones derivadas de la misma; las principales son la insuficiencia valvular aórtica, el aneurisma aórtico sifilítico y la estenosis del ostium de las coronarias. La más frecuente es la insuficiencia aórtica, que 


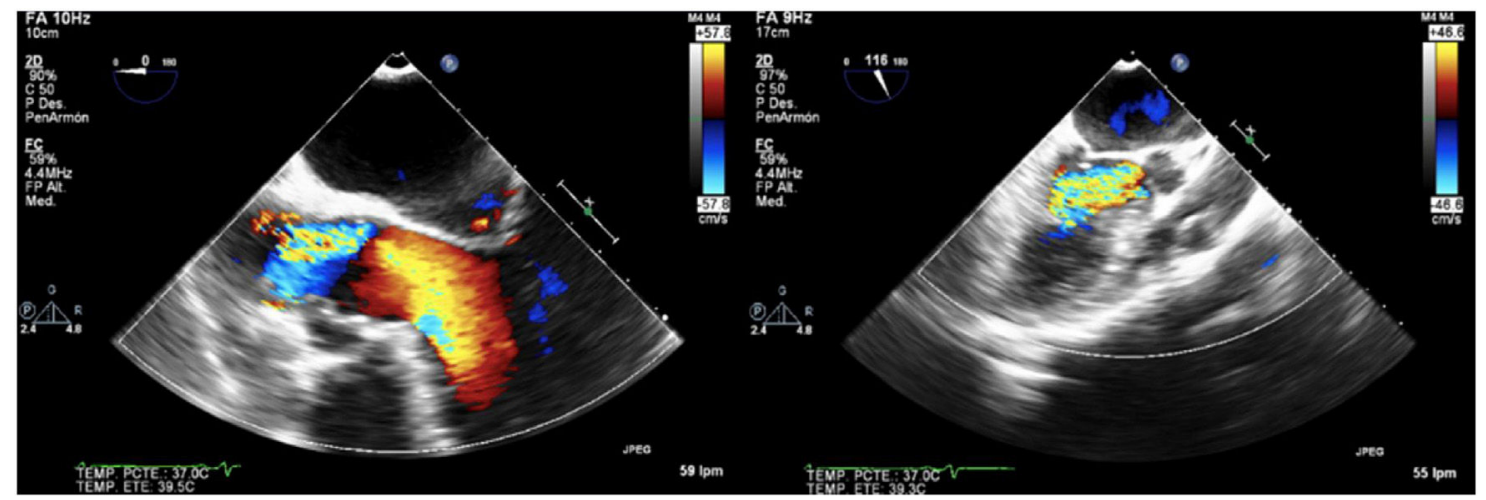

Figura 1 Imagen tomada durante ecocardiografía transesofágica en la cual se evidencia turbulencia del flujo en la válvula aórtica y el tracto de salida del ventrículo izquierdo, explicada por insuficiencia aórtica severa.

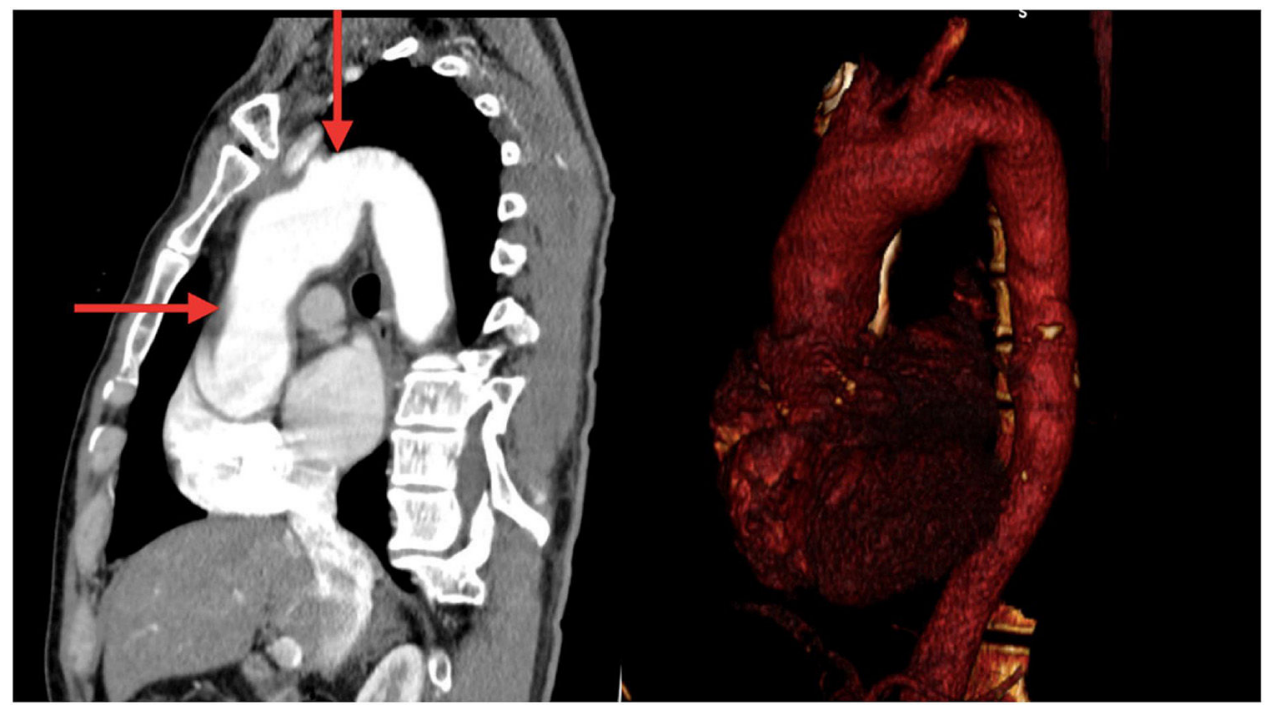

Figura 2 Vista tomográfica y reconstrucción $3 \mathrm{D}$ que evidencia dilatación aneurismática de la aorta ascendente. Las flechas rojas delimitan la extensión de la misma. El aneurisma fue de $45 \mathrm{~mm}$ de diámetro y, por tanto, no requirió corrección.

se da en el $20 \%$ al $30 \%$ de los pacientes y es secundaria a dilatación de la raiz aórtica, adelgazamiento del anillo valvular y deformación de las valvas. Al igual que en la insuficiencia aórtica por otras etiologías, hay síntomas de falla cardíaca y es frecuente auscultar un soplo diastólico más audible en el borde esternal derecho ${ }^{3}$.

El aneurisma sifilítico es tres veces menos frecuente que la insuficiencia aórtica, detectándose en el 5 al 10\% de los pacientes; suele ser sacular y deberse a la cicatrización difusa de la pared aórtica; en general, no es propenso a la disección ${ }^{6}$. Se ubica comúnmente en la aorta ascendente (50\%), seguido del arco aórtico (35\%) y la aorta descendente $(15 \%)^{11}$. Su manifestación clínica es variable y depende de la estructura que comprima; puede haber dolor torácico, disnea, tos, obstrucción venosa o arterial o incluso masa supraclavicular. En la mayoría de casos son asintomáticos y se encuentran de forma incidental ${ }^{3}$. Aunque poco frecuente, en estadios avanzados puede haber complicaciones fatales como disección o ruptura.

La estenosis del ostium coronario es la menos frecuente de las complicaciones de la aortitis sifilítica ${ }^{6}$. Es común que los pacientes refieran síntomas de angina. El infarto del miocardio es raro, y probablemente se explique por el proceso lento y progresivo de la estenosis que permite el desarrollo de circulación colateral ${ }^{3}$.

Hasta el $43 \%$ de los pacientes con sífilis cardiovascular tienen neurolúes asociada ${ }^{12}$. Las manifestaciones neurológicas por compromiso sifilítico meningovascular pueden ser vértigo, cambios en la personalidad, convulsiones y accidentes cerebrovasculares ${ }^{13}$; esta última es la manifestación con la que debutó el paciente del caso. En consecuencia, en los pacientes con sífilis cardiovascular es imperativo realizar estudio de líquido cefalorraquídeo para definir el esquema antibiótico más apropiado.

De otro lado, el diagnóstico de sífilis cardiovascular se basa en criterios serológicos, histológicos y de imagen. Siempre se debe sospechar en pacientes con dilatación aneurismática de la porción proximal de la aorta y calcificación linear de su pared anterolateral. La prueba inicial debe ser no treponémica y confirmar con treponémica. El estudio histológico de la aorta usualmente revela engrosamiento fibroso de la adventicia con pérdida de la vasa vasorum, rodeado de células linfoplasmocitarias y pérdida de las fibras de elastina en la media 


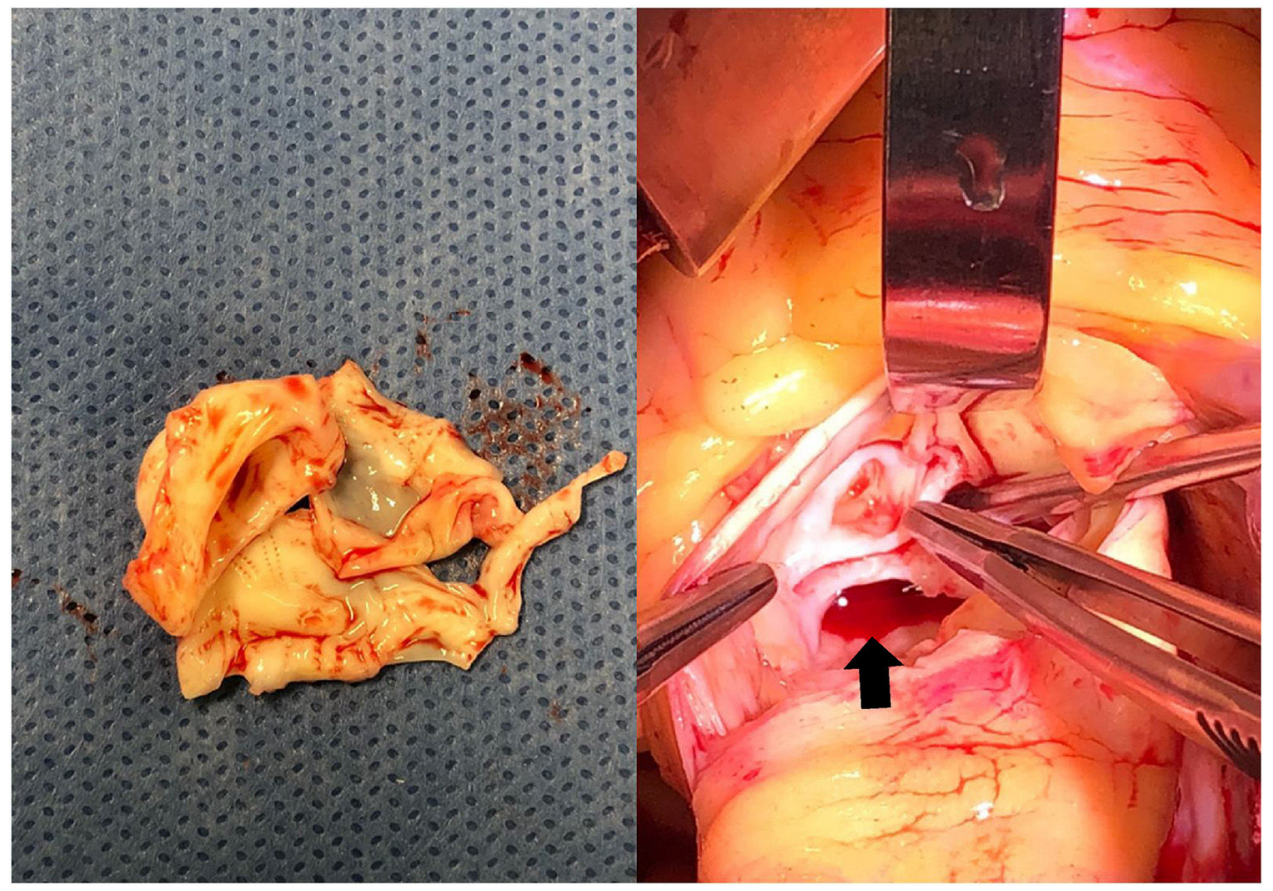

Figura 3 Imagen en la que se evidencia resección de válvula aórtica trivalva para posterior recambio valvular aórtico. Con flecha de color negro se resalta la perforación de la valva coronariana derecha.

con engrosamiento aterosclerótico de la íntima. No se han encontrado espiroquetas dentro de la aorta ${ }^{4}$.

En cuanto al tratamiento antibiótico, el Treponema pallidum conserva adecuada sensibilidad a la penicilina y por tanto ésta continúa siendo la primera línea terapéutica. En el caso de sífilis cardiovascular, debe darse penicilina benzatínica 2.4 millones de unidades intramusculares cada 7 días por 3 dosis. Si hay compromiso del sistema nervioso asociado, el tratamiento se basa en penicilina cristalina por 10 a 14 días. ${ }^{14}$

\section{Conclusión}

La sífilis continúa siendo una infección prevalente en nuestra población, de modo que es crucial conocer sus diferentes manifestaciones clínicas. Las formas cardiovasculares más frecuentes son la insuficiencia valvular aórtica, el aneurisma de aorta ascendente y la obstrucción del ostium de las coronarias, las cuales se evidencian de forma tardía en el curso de la enfermedad, de ahí que puedan constituir un reto diagnóstico. Es imprescindible descartar compromiso concomitante del sistema nervioso central.

\section{Financiación}

Ninguna.

\section{Conflicto de intereses}

Ninguno.

\section{Bibliografía}

1. WHO | Prevalence and incidence of selected sexually transmitted infections [Internet]. WHO. [Acceso 6 Nov 2018]. Disponible en: http://www.who.int/ reproductivehealth/publications/rtis/9789241502450/en/.

2. Gjestland T. The Oslo study of untreated syphilis; an epidemiologic investigation of the natural course of the syphilitic infection based upon a re-study of the Boeck-Bruusgaard material. Acta Derm Venereol Suppl (Stockh). 1955;35 Suppl 34:3-368. Annex I-LVI.

3. Jackman JD, Radolf JD. Cardiovascular syphilis. Am J Med. 1989;87:425-33.

4. Byard RW. Syphilis-cardiovascular manifestations of the great imitator. J Forensic Sci. 2018;63:1312-5.

5. Roberts WC, Ko JM, Vowels TJ. Natural history of syphilitic aortitis. Am J Cardiol. 2009;104:1578-87.

6. Radolf JD, Tramont EC, Salazar JC. Sífilis (Treponema pallidum) En: Enfermedades infecciosas. Principios y práctica. Elsevier. 2016:2832-59.

7. Graciaa DS, Mosunjac MB, Workowski KA, Kempker RR. Asymptomatic cardiovascular syphilis with aortic regurgitation requiring surgical repair in an HIV-infected patient. Open Forum Infect Dis [Internet]. 2017;4(4.) [Acceso 6 Nov 2018] Disponible en: https://academic.oup.com/ofid/article/4/4/ofx198/4158427.

8. Situación de las infecciones de transmision1.pdf [Internet]. [Acceso 6 Nov 2018]; Disponible en: https://www. minsalud.gov.co/salud/Documents/observatorio_vih/ documentos/monitoreo_evaluacion/1_vigilancia_salud_ publica/a_situacion_epidimiologica/SITUACION\%20DE\%20LAS\% 20INFECCIONES\%20DE\%20TRANSMISION1.pdf.

9. Stone JR, Bruneval P, Angelini A, Bartolini G, Basso C, Batoroeva $\mathrm{L}$, et al. Consensus statement on surgical pathology of the aorta from the Society for Cardiovascular Pathology and the Association for European Cardiovascular Pathology: I Inflammatory diseases. Cardiovasc Pathol J Soc Cardiovasc Pathol. 2015;24:267-78. 
10. Miller DV, Isotalo PA, Weyand CM, Edwards WD, Aubry MC, Tazelaar HD. Surgical pathology of noninfectious ascending aortitis: a study of 45 cases with emphasis on an isolated variant. Am J Surg Pathol. 2006;30:1150-8.

11. Yuan S-M. Syphilitic aortic aneurysm. Z Für Rheumatol. 2018;77:741-8.

12. Singh AE, Romanowski B. Syphilis: review with emphasis on clinical, epidemiologic, and some biologic features. Clin Microbiol Rev. 1999;12:187-209.
13. Classical syphilitic lesions from the museum | SpringerLink [Internet]. [Acceso 27 Mar 2019]; Disponible en: https://link.springer.com/article/10.1007\%2Fs12024-0180012-9.

14. Hook EW. Syphilis. The Lancet. 2017;389(10078):1550-7. 